\title{
Direct Observation of Ultrafast Exciton Formation in a Monolayer of $\mathrm{WSe}_{2}$
}

\author{
Philipp Steinleitner, ${ }^{\circledR}$ Philipp Merkl, Philipp Nagler, Joshua Mornhinweg, Christian Schüller, Tobias Korn, \\ Alexey Chernikov,* and Rupert Huber* \\ Department of Physics, University of Regensburg, 93040 Regensburg, Germany
}

\section{Supporting Information}

ABSTRACT: Many of the fundamental optical and electronic properties of atomically thin transition metal dichalcogenides are dominated by strong Coulomb interactions between electrons and holes, forming tightly bound atom-like states called excitons. Here, we directly trace the ultrafast formation of excitons by monitoring the absolute densities of bound and unbound electron-hole pairs in single monolayers of $\mathrm{WSe}_{2}$ on a diamond substrate following femtosecond nonresonant optical excitation. To this end, phaselocked mid-infrared probe pulses and field-sensitive electro-optic sampling are used to map out the full complex-valued optical conductivity of the nonequilibrium system and to discern the hallmark low-energy responses of bound and unbound pairs. While the spectral shape of the infrared response immediately after above-bandgap injection is

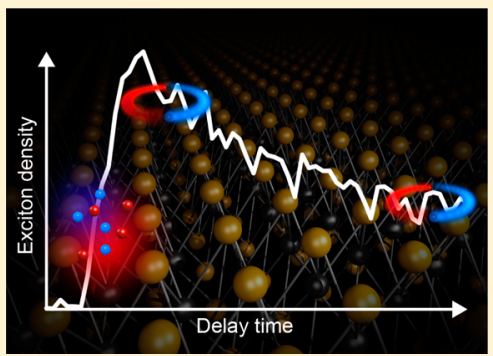
dominated by free charge carriers, up to $60 \%$ of the electron-hole pairs are bound into excitons already on a subpicosecond time scale, evidencing extremely fast and efficient exciton formation. During the subsequent recombination phase, we still find a large density of free carriers in addition to excitons, indicating a nonequilibrium state of the photoexcited electron-hole system.

KEYWORDS: Dichalcogenides, atomically thin $2 \mathrm{D}$ crystals, exciton formation, ultrafast dynamics

A tomically thin transition metal dichalcogenides (TMDCs) have attracted tremendous attention due to their direct bandgaps in the visible spectral range, ${ }^{1,2}$ strong interband optical absorption, ${ }^{3,4}$ intriguing spin-valley physics, ${ }^{5-7}$ and applications as optoelectronic devices. ${ }^{8-11}$ The physics of twodimensional (2D) TMDCs are governed by strong Coulomb interactions owing to the strict quantum confinement in the out-of-plane direction and the weak dielectric screening of the environment. ${ }^{12,13}$ Electrons and holes in these materials can form excitons with unusually large binding energies of many hundreds of millielectronvolts, ${ }^{14-19}$ making these quasiparticles stable even at elevated temperatures and high carrier densities. $^{20,21}$ The properties of excitons in 2D TMDCs are a topic of intense research, investigating, for example, rapid exciton-exciton scattering, ${ }^{22}$ interlayer excitons, ${ }^{23}$ charged excitons and excitonic molecules, ${ }^{24,25}$ ultrafast recombination dynamics, ${ }^{19,26-28}$ or efficient coupling to light and lattice vibrations. ${ }^{4,19,29,30}$

In many experiments, excitons are created indirectly through nonresonant optical excitation or electronic injection, which may prepare unbound charge carriers with energies far above the exciton resonance. ${ }^{8,18}$ Subsequently, the electrons and holes are expected to relax toward their respective band minima and form excitons in the vicinity of the fundamental energy gap. In principle, strong Coulomb attraction in 2D TMDCs should foster rapid exciton formation. Recent optical pump-probe studies relying on interband transitions have reported characteristic formation times on subpicosecond time-scales. ${ }^{31}$ The relaxation of large excess energies, however, requires many scattering processes, which can lead to nonequilibrium carrier and phonon distributions and, potentially, to a mixture of excitons and unbound electron-hole pairs. Hence the question of how excitons and free charge carriers evolve after abovebandgap excitation is of central importance for the fundamental understanding of 2D TMDCs.

Quantifying the densities of bound and unbound carrier populations has remained challenging for optical interband spectroscopy, because both species tend to induce similar modifications in the interband response. Conversely, terahertz and mid-infrared (mid-IR) probes may sensitively discriminate between bound and unbound states via characteristic spectral fingerprints of their low-energy elementary excitations. ${ }^{19,32-37}$ As schematically illustrated in Figure 1a, excitons efficiently absorb radiation in the spectral range of the intraexcitonic resonances, corresponding to dipole-allowed transitions from the exciton ground state (1s) to higher excited states $(2 p, 3 p$, $4 \mathrm{p} . .$.$) , labeled in analogy to the hydrogen series. This$ absorption occurs irrespective of the exciton center-of-mass momentum and interband optical selection rules. ${ }^{19,33-38}$ In contrast, free electrons and holes feature a more inductive Drude-like response. ${ }^{32,33}$ By using ultrabroadband electro-optic detection of the waveform of a few-cycle mid-IR probe pulse before and after the excitation of the electron-hole system, the energy-resolved intraband response, characterized by the real and imaginary part of the complex conductivity, can be traced on the femtosecond scale without resorting to a Kramers-

Received: October 21, 2016

Revised: December 21, 2016

Published: February 9, 2017 
(a)

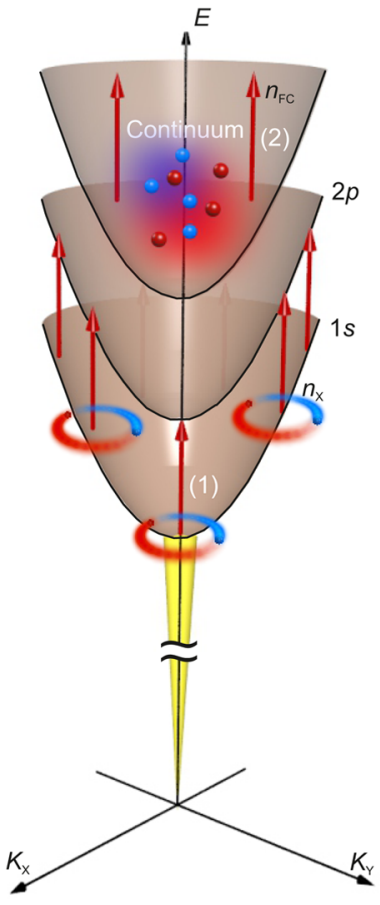

(b)

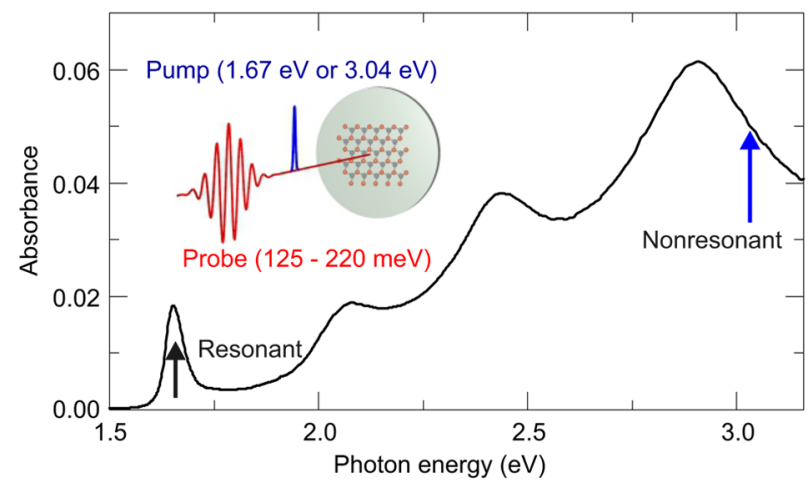

(c)

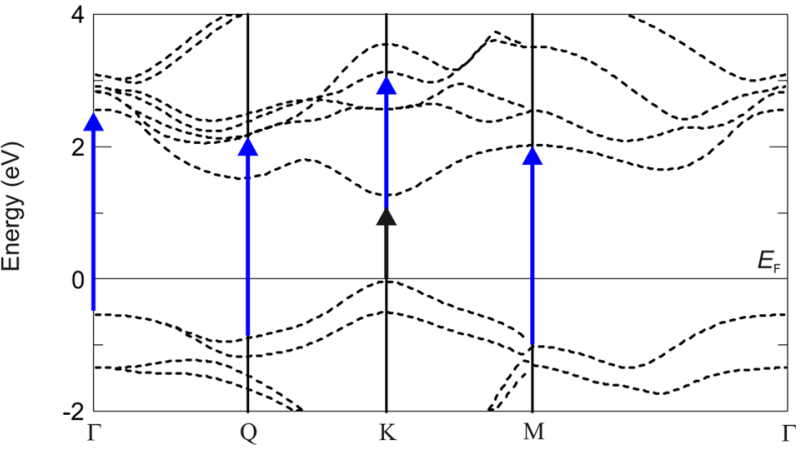

Figure 1. (a) Schematic illustration of the low-energy response of excitons and unbound electron-hole pairs. The dispersion relations are shown in the two-particle picture, corresponding to exciton states with the principal quantum numbers $n=1,2$, 3, and so forth, and the electron-hole continuum (topmost band), presented as a function of the center-of-mass momentum $K$. The yellow-shaded area represents the region of the light cone, where the excitons can be directly excited by photons due to momentum conservation. Red arrows schematically indicate relevant intraband excitation processes: (1) dipole-allowed transition between $1 \mathrm{~s}$ and $2 \mathrm{p}$ excitons; $n_{\mathrm{X}}$ denotes the 1s population; (2) off-resonant excitation of unbound electron-hole pairs of density $n_{\mathrm{FC}}$ (b) Typical optical interband absorption spectrum (black solid line) of the WSe ${ }_{2}$ monolayer, see ref 41 . The black arrow marks the photon energy of $1.67 \mathrm{eV}$ for resonant carrier injection, whereas the blue arrow denotes the photon energy used for nonresonant excitation at $3.04 \mathrm{eV}$. Inset: Schematic of the femtosecond optical-pump/mid-IR-probe experiment of single-layer WSe $\mathrm{H}_{2}$ on a diamond substrate. The mid-IR probe pulse (red) is delayed with respect to the optical pump pulse (blue) by a variable time delay. The electric field of the probe is subsequently detected as a function of the electro-optic sampling delay time. (c) Calculated band structure of the WSe $e_{2}$ monolayer from ref 42 . The black arrow highlights the fundamental transition for resonant carrier injection. A few possible transitions in case of the nonresonant excitation are roughly sketched by blue arrows.

Kronig analysis. ${ }^{32}$ Drude and Lorentzian signatures from unbound carriers and excitons, respectively, have been previously identified in bulk $\mathrm{Cu}_{2} \mathrm{O}^{34,38,39}$ and $\mathrm{GaAs}$ quantum wells, ${ }^{33-36}$ among other material systems. Recently, this technique has become sensitive enough to probe the intraexcitonic transitions even in single atomically thin layers of TMDCs following direct optical injection of the excitons. ${ }^{19,37}$ The exciton formation, however, as well as the dynamics of unbound photoexcited charge carriers in a $2 \mathrm{D}$ TMDC has not been studied by direct low-energy probing so far, to the best of our knowledge.

Here, we employ field-sensitive mid-IR femtosecond probing to directly monitor the dynamics of photoexcited electronhole pairs in the prototypical single-layer 2D TMDC $\mathrm{WSe}_{2}$. After highly nonresonant (excess energy $\sim 1.4 \mathrm{eV}$ ) femtosecond interband excitation, the complex-valued mid-IR conductivity indicates a rapid carrier relaxation toward the respective band minima during the first hundreds of femtoseconds. Remarkably, more than half of the carriers are bound into excitons already 0.4 ps after the excitation. The ratio between excitons and unbound electron-hole pairs increases slightly in the subsequent $0.4 \mathrm{ps}$ and both populations decay on a time scale of a few picoseconds while a significant fraction of free carriers is still observed after 5 ps, strongly indicating highly nonequilibrium conditions of the electron-hole system.
Our samples were prepared by mechanical exfoliation of $\mathrm{WSe}_{2}$ bulk crystals (commercially acquired from HQgraphene) on viscoelastic substrates and were subsequently transferred onto diamond. ${ }^{40}$ Monolayer flakes with typical diameters of the order of $100 \mu \mathrm{m}$ were identified by photoluminescence and reflectance contrast spectroscopy. An overview of the basic experimental concept is schematically illustrated in the inset of Figure $1 \mathrm{~b}$. The samples were optically excited with $100 \mathrm{fs}$ laser pulses (repetition rate: $0.4 \mathrm{MHz}$ ) centered at a photon energy of either $1.67 \mathrm{eV}$, for resonant creation of the $1 \mathrm{~s} \mathrm{~A}$ exciton of $\mathrm{WSe}_{2}$, or $3.04 \mathrm{eV}$, for excitation far above the fundamental bandgap (see Supporting Information Figure S1). The pump fluence was set to 19 or $38 \mu \mathrm{J} / \mathrm{cm}^{2}$ as detailed below. As a probe, we used a phase-locked mid-IR pulse (duration: $50 \mathrm{fs}$, fwhm), generated by optical rectification of the fundamental laser output in a $50 \mu \mathrm{m}$ thick $\mathrm{AgGaS}_{2}$ crystal (see Supporting Information Figure S2). The probe spectrum covers a frequency window between 30 and $53 \mathrm{THz}$, encompassing the energy of the $1 \mathrm{~s}-2 \mathrm{p}$ intraexcitonic transition of $\mathrm{WSe}_{2}$ monolayers at $\sim 170 \mathrm{meV}$ (corresponding to a frequency of $41 \mathrm{THz}) .{ }^{19}$ For a given delay time $t_{\mathrm{PP}}$ between pumping and probing, the complete electric field waveform $E_{\mathrm{ref}}\left(t_{\mathrm{EOS}}\right)$ of the transmitted probe pulse in absence of excitation as well as its pump-induced change $\Delta E\left(t_{\mathrm{EOS}}, t_{\mathrm{PP}}\right)$ was recorded electrooptically as a function of the detection time $t_{\mathrm{EOS}}$ (see 
Supporting Information Figure S3). All experiments were performed at room temperature and ambient conditions. We note that the illumination of the sample with a photon energy of $3.04 \mathrm{eV}$ introduced additional broadening and a small redshift of the exciton resonance. The exposure time was thus carefully chosen such that the characteristic intraexcitonic fingerprint was still observed at resonant excitation conditions after illumination with $3.04 \mathrm{eV}$ photons (see Supporting Information Figure S5 and S6 together with discussion).

In the first set of experiments, we tuned the pump photon energy to the interband absorption maximum corresponding to the $1 \mathrm{~s}$ state of the A exciton ${ }^{41}$ (Figure 1b, black arrow). The corresponding single-particle transitions occur at the $\mathrm{K}$ and $\mathrm{K}^{\prime}$ points of the Brillouin zone ${ }^{42}$ (Figure 1c). Figure 2a (upper (a)

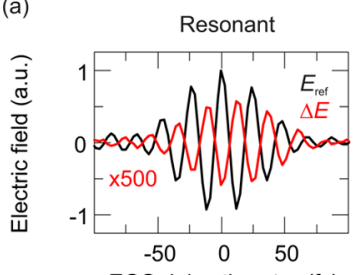

EOS delay time $t_{\text {EOS }}$ (fs)

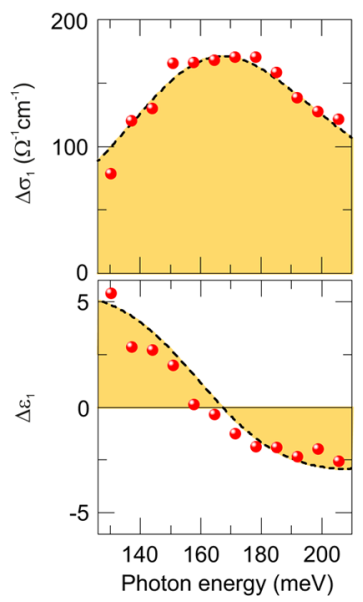

(b)

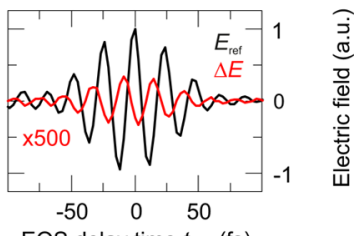

EOS delay time $t_{\mathrm{EOS}}(\mathrm{fs})$

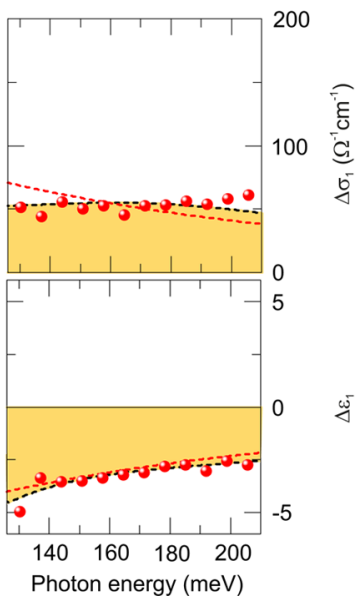

Figure 2. (a) Upper panel: time-resolved waveforms of the probe pulse $E_{\text {ref }}$ transmitted through the $\mathrm{WSe}_{2}$ monolayer in the absence of excitation (black curve) and the pump-induced change $\Delta E$ (red curve, scaled up by a factor of 500) after resonant excitation $\left(t_{\mathrm{pp}}=75 \mathrm{fs}\right.$, pump fluence: $19 \mu \mathrm{J} / \mathrm{cm}^{2}$ ) in ambient conditions. Lower panel: corresponding pump-induced changes of the real parts of the optical conductivity $\Delta \sigma_{1}$ (top) and the dielectric function $\Delta \varepsilon_{1}$ (bottom) as a function of the photon energy. The experimental data is shown by red circles; the results from the Drude-Lorentz model are plotted as black-dashed lines with shaded areas. (b) Same as (a) for nonresonant excitation $\left(t_{\mathrm{PP}}=400 \mathrm{fs}\right.$, pump fluence: $\left.19 \mu \mathrm{J} / \mathrm{cm}^{2}\right)$. The lower panel includes a fit by the Drude model (i.e., excluding excitons), indicated by the red-dashed line.

panel) shows the waveform of the transmitted probe pulse $E_{\text {ref }}\left(t_{\mathrm{EOS}}\right)$ in absence of excitation (black curve) together with the pump-induced change $\Delta E\left(t_{\mathrm{EOS}}, t_{\mathrm{PP}}=75 \mathrm{fs}\right)$ (red curve). The observed phase shift of $\Delta E$ with respect to $E_{\text {ref }}$ of exactly $\pi$ is already a hallmark of a dominantly absorptive response, as expected in the case of purely excitonic population. ${ }^{19,43}$ This assignment is corroborated by the extracted changes in the real parts of the mid-IR conductivity $\left(\Delta \sigma_{1}\right)$ and the dielectric function $\left(\Delta \varepsilon_{1}\right)$, presented in Figure 2a (lower panel) roughly corresponding to absorptive and inductive components, respectively. In particular, a broad peak in $\Delta \sigma_{1}$, centered at a photon energy of $\hbar \omega=170 \mathrm{meV}$, combined with a dispersive shape of $\Delta \varepsilon_{1}$, crossing the zero-axis at the same energy, are characteristic of the intraexcitonic $1 s-2 p$ resonance in $\mathrm{WSe}_{2}$, as discussed in detail in ref 19.

A qualitatively different picture is obtained for nonresonant excitation. The pump photon energy of $3.04 \mathrm{eV}$ (Figure $1 \mathrm{~b}$, blue arrow) allows for carrier injection into higher-lying states of the $\mathrm{WSe}_{2}$ monolayer with a variety of possible electronic transitions across the Brillouin zone, as schematically illustrated in Figure 1c. This broad energy region is often chosen for the excitation in optical experiments due to the high absorption of the TMDC materials ${ }^{41}$ and the availability of commercial laser sources in this spectral range. The differences to the situation after resonant excitation are already apparent in the electric field trace of the probe pulse, presented in Figure $2 \mathrm{~b}$ (upper panel). The relative phase of $\Delta E\left(t_{\mathrm{EOS}}, t_{\mathrm{PP}}=400 \mathrm{fs}\right)$ deviates strongly from $\pi$ and is closer to $\pi / 2$, indicating the predominantly inductive response of an electron-hole plasma. ${ }^{43}$ The shape of the corresponding spectral features (Figure 2b, lower panel) is in stark contrast to the observations for resonant injection. Instead of a peak, $\Delta \sigma_{1}$ is now spectrally flat, accompanied by a negative, monotonically increasing $\Delta \varepsilon_{1}$ across the experimentally accessible energy range. This shape is characteristic of the Drude-like behavior of free charge carriers. $^{32,33,36,43}$

For a quantitative analysis of the measured spectra, we apply a phenomenological Drude-Lorentz model. ${ }^{36,43}$ Within this approach, pump-induced changes in the frequency-dependent dielectric function $\Delta \varepsilon(\omega)=\Delta \varepsilon_{1}+i \Delta \sigma_{1} /\left(\varepsilon_{0} \omega\right)$ are described using two components

$$
\Delta \varepsilon(\omega)=\frac{n_{\mathrm{X}} e^{2}}{d \varepsilon_{0} \mu} \times \frac{f_{1 \mathrm{~s}, 2 \mathrm{p}}}{\frac{E_{\mathrm{res}}^{2}}{\hbar^{2}}-\omega^{2}-i \omega \Delta}-\frac{n_{\mathrm{FC}} e^{2}}{d \varepsilon_{0} \mu} \times \frac{1}{\omega^{2}+i \omega \Gamma}
$$

The first term, a Lorentzian resonance, accounts for the intraexcitonic $1 \mathrm{~s}-2 \mathrm{p}$ absorption. It includes the $1 s$ exciton density $n_{\mathrm{X}}$, the corresponding reduced mass $\mu=m_{\mathrm{e}} m_{\mathrm{h}} /\left(m_{\mathrm{e}}+\right.$ $\left.m_{\mathrm{h}}\right)$, obtained from the effective masses $m_{\mathrm{e}}$ and $m_{\mathrm{h}}$ of the constituting electron and hole, the effective thickness $d$ of the monolayer (treated as a thin slab in this model), the oscillator strength $f_{1 \mathrm{~s}, 2 \mathrm{p}}$ of the intraexcitonic transition, the resonance energy $E_{\text {res }}$, and the line width $\Delta$. Additional constants are the electron charge $e$ and the vacuum permeability $\varepsilon_{0}$. The second term represents the Drude response of the electron-hole plasma, which depends on the pair density of free carriers $n_{\mathrm{FC}}$ and their scattering rate $\Gamma$. For the analysis of the data, we fix the reduced mass $\mu=0.17 \mathrm{~m}_{0}^{12}$ and the oscillator strength $f_{1 \mathrm{~s}, 2 \mathrm{p}}$ $=0.32,{ }^{19}$ corresponding to electron-hole properties at the $\mathrm{K}$ and $\mathrm{K}^{\prime}$ valleys in $\mathrm{WSe}_{2}$, and set the effective layer thickness $d$ to $0.7 \mathrm{~nm}$. The remaining parameters of the Drude-Lorentz model $\left(n_{\mathrm{X}}, n_{\mathrm{FC}}, E_{\mathrm{res}}, \Delta, \Gamma\right)$ are then extracted by fitting the experimental data. Note that the fact that both independently measured $\Delta \sigma_{1}$ and $\Delta \varepsilon_{1}$ spectra need to be simultaneously reproduced poses strict limits to the possible values of the fitting parameters. The numerical adaptation (Figure 2a,b, black dashed curves) yields an overall good fit quality, allowing for a meaningful extraction of the parameters. We further note that the extracted exciton and free carrier densities are obtained as absolute quantities for a given choice of material parameters, such as carrier effective masses and the oscillator strength, 
without the requirement of any a priori assumptions of the density ratios.

For resonant excitation, we find an exciton density of $n_{\mathrm{X}}=$ $3.4 \times 10^{12} \mathrm{~cm}^{-2}$ and, as expected, virtually no contribution from unbound electron-hole pairs. The $1 \mathrm{~s}-2 \mathrm{p}$ resonance energy of $167 \mathrm{meV}$ and the peak line width of $99 \mathrm{meV}$ also compare well with previous observations. ${ }^{19}$ Conversely, the response after nonresonant excitation includes a significant fraction of unbound electron-hole pairs $n_{\mathrm{FC}}$ of $0.8 \times 10^{12} \mathrm{~cm}^{-2}$ (with $n_{\mathrm{X}}=1.5 \times 10^{12} \mathrm{~cm}^{-2}, E_{\text {res }}=180 \mathrm{meV}, \Delta=182 \mathrm{meV}$, and $\Gamma=$ $\left.0.052 \mathrm{fs}^{-1}\right)$. Whereas the qualitative shape of the measured response functions in Figure $2 \mathrm{~b}$ can be roughly accounted for by the Drude model alone (red dotted lines), the twocomponent model (see eq 1) reproduces the data more accurately. This observation already indicates that electronhole correlations are important even for delay times as short as $t_{\mathrm{PP}}<1$ ps.

The ultrafast evolution of the mid-IR of a nonresonantly generated electron-hole system response is systematically shown in Figure 3, which displays spectra of $\Delta \sigma_{1}$ and $\Delta \varepsilon_{1}$ for a series of delay times $t_{\mathrm{PP}}$ together with the fit curves from the Drude-Lorentz model. The corresponding field-resolved timedomain data are given in Supporting Information Figure S7. At $t_{\mathrm{PP}}=0 \mathrm{fs}$, we recover a predominantly Drude-like response, as previously discussed. After $400 \mathrm{fs}$, the overall magnitude of the

(a)

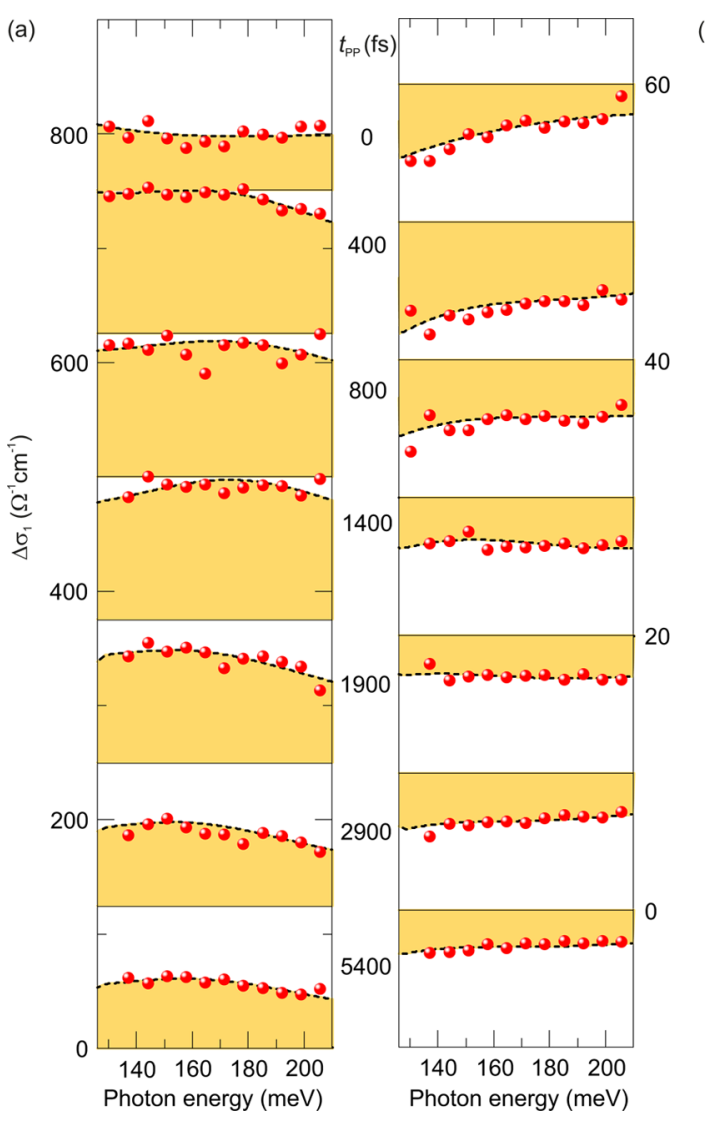

Figure 3. Pump-induced changes of the real parts of the optical conductivity $\Delta \sigma_{1}$ (a) and the dielectric function $\Delta \varepsilon_{1}$ (b) of the photoexcited $\mathrm{WSe}_{2}$ monolayer as a function of the photon energy for several pump delays $t_{\mathrm{pp}}$ after nonresonant excitation. The pump fluence is set to $38 \mu \mathrm{J} / \mathrm{cm}^{2}$. Red spheres denote the experimental data and the black dashed curves represent the results by the DrudeLorentz model fitting simultaneously $\Delta \sigma_{1}$ and $\Delta \varepsilon_{1}$. signal increases and a broad resonance centered at about 160 $\mathrm{meV}$ develops. On the basis of the close correspondence to the $1 s-2 p$ absorption measured under resonant excitation conditions, this resonance is attributed to the rising exciton population. For $t_{\mathrm{PP}}>0.8 \mathrm{ps}$, all pump-induced changes decrease, indicating carrier recombination. More importantly, the measured response remains characteristic of a mixture of excitons and free carriers at all delay times. In particular, we observe a broad excitonic feature in $\Delta \sigma_{1}$ but also a flat negative response in $\Delta \varepsilon_{1}$ from the electron-hole plasma across all spectra.

The densities of bound and unbound electron-hole pairs, extracted from the Drude-Lorentz fit, are presented in Figure 4

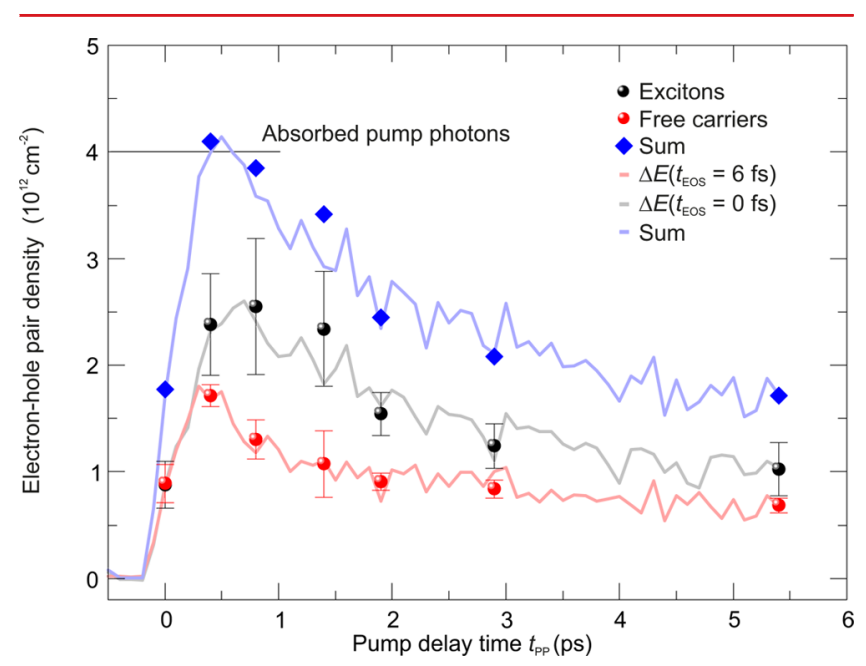

Figure 4. Absolute electron-hole pair densities extracted from the data shown in Figure 3, using the Drude-Lorentz model, including 1s excitons $n_{\mathrm{X}}$ (black spheres), unbound electron-hole pairs $n_{\mathrm{FC}}$ (red spheres), and the total density of the two contributions (blue diamonds) as a function of the delay time $t_{\mathrm{pp}}$ after nonresonant excitation. The error bars represent the $95 \%$ confidence intervals of the fitting parameters. In addition, pump-induced changes $\Delta E$ recorded at fixed electro-optic sampling times of $t_{\mathrm{EOS}}=0 \mathrm{fs}$ (gray solid line) and 6 fs (red solid line) as a function of $t_{\mathrm{PP}}$ are presented. These changes are proportional to the exciton and plasma densities, respectively, as discussed in the main text. The horizontal solid line marks the number of absorbed pump photons as estimated from the applied pump fluence and the optical absorbance.

(spheres) as a function of $t_{\mathrm{PP}}$ together with the total pair density, $n_{\text {tot }}=n_{\mathrm{X}}+n_{\mathrm{FC}}$. Interestingly, the extracted densities reach their respective maxima at finite delays of $t_{\mathrm{PP}}=0.4 \mathrm{ps}$ for $n_{\mathrm{FC}}$ and $n_{\text {tot }}$ and $t_{\mathrm{PP}}=0.8$ ps for $n_{\mathrm{X}}$. All densities subsequently decay on a few-picoseconds scale. For a consistency check, we also measured the transient changes of the electric field $\Delta E\left(t_{\mathrm{PP}}\right)$ at fixed electro-optic delays of $t_{\mathrm{EOS}}=0$ and $6 \mathrm{fs}$. Because these times correspond to phase-shifts of $\pi$ and $\pi / 2$ with respect to the peak of the waveform $E_{\text {ref }}$ (Figure 2a,b), the $\Delta E$ signals at these delays should be mostly sensitive to exciton and plasma densities, respectively, as discussed above (see also Supporting Information Figure S4 together with discussion). Indeed, these data (Figure 4, solid curves) almost perfectly trace the individual dynamics of excitons and plasma obtained from the full spectral analysis (Figure 4, spheres). The temporal evolution of the remaining fitting parameters $\left(\Delta, E_{\text {res }}, \Gamma\right)$ is given in Supporting Information Figure S8.

Our experimental findings provide important insights into key aspects of the microscopic dynamics of the photogenerated 
electron-hole pairs. We start out by discussing the delayed rise of the carrier densities with respect to the pump pulse, which we attribute to ultrafast carrier relaxation. As indicated in Figure $1 c$, the carriers are injected into comparatively flat sections of the electronic band structure, defining states with large effective masses. As we model the mid-IR response using the reduced mass from the band edge $\mathrm{K}$ (and $\mathrm{K}^{\prime}$ ) states, the extracted carrier density underestimates the actual number of carriers at these early delay times. This argument is quantitatively consistent with an estimate of the injected carrier density by taking into account an effective optical absorption of the diamond-supported $\mathrm{WSe}_{2}$ monolayer of about $5 \%$ at a photon energy of $3.04 \mathrm{eV}$. As shown in Figure 4, the number of absorbed photons $\left(4 \times 10^{12} \mathrm{~cm}^{-2}\right)$ closely matches the maximum measured density $n_{\text {tot }}=n_{\mathrm{X}}+n_{\mathrm{FC}}$ of bound and unbound electron-hole pairs extracted from the experiment. Thus, at $t_{\mathrm{PP}}=0.4 \mathrm{ps}$, we can quantitatively account for every absorbed photon resulting in an electron-hole pair residing close to the band edges of $\mathrm{WSe}_{2}$.

Second, for $t_{\mathrm{PP}}=0.4 \mathrm{ps}$, roughly $60 \%$ of the injected carriers are found to be already bound into excitons. Until $t_{\mathrm{pP}}=0.8 \mathrm{ps}$, additional excitons are formed from free charge carriers and the exciton fraction reaches its maximum of about $70 \%$ while the plasma density decreases. The exciton formation in monolayer $\mathrm{WSe}_{2}$ is thus significantly faster, by about 2 orders of magnitude, than the corresponding process in GaAs quantum wells. ${ }^{33,43}$ It is also more rapid than the recently reported trion formation in monolayer TMDCs, which evolves on the time scale of several picoseconds. ${ }^{44}$ Subsequently, both excitons and unbound carriers decay through radiative and nonradiative recombination. Remarkably, the fraction of bound and unbound carriers remains roughly constant during the carrier lifetime up to the maximum studied time delay of $5.4 \mathrm{ps}$. By assuming a thermal population and using the Saha equation, ${ }^{43}$ the corresponding effective carrier temperature is estimated to be of the order of $1500 \mathrm{~K}$. Consequently, the exciton and plasma populations are far from equilibrium considering the lattice temperature of $295 \mathrm{~K}$. This scenario appears reasonable considering the high initial excess energy and the short carrier lifetime of a few picoseconds. At these conditions, fast initial carrier relaxation could result in nonequilibrium phonon populations subsequently keeping the effective carrier temperature high through reabsorption, the so-called hot-phonon effect. ${ }^{45}$ An alternative explanation of reaching a bottleneck in electron-phonon scattering by phase-space restrictions appears less likely, because the estimated temperature of $1500 \mathrm{~K}$ is well above typical optical and acoustic zone-edge phonon energies in $\mathrm{WSe}_{2}{ }^{46}$ and should thus not impede further relaxation through phonon emission.

Finally, we note that excitons can be present both as optically bright, spin-allowed $\mathrm{K}-\mathrm{K}$ excitons (labeled by the location of the corresponding interband transitions in the Brillouin zone) and as spin- and momentum-forbidden dark states. In addition, relaxation of the exciton population toward intervalley $\mathrm{K}-\mathrm{K}^{\prime 47}$ and $\mathrm{K}-\mathrm{Q} / \Sigma$ states $^{30}$ is possible. However, due to the similar binding energies of the different states and the broadening of the measured $1 s-2 p$ resonance, the excitons in our experiment should be generally considered as a mixture of these configurations. Because of the relatively small variation of effective exciton masses, however, ranging from $0.16 \mathrm{~m}_{0}$ (bright $\mathrm{K}-\mathrm{K}$ excitons) to $0.23 \mathrm{~m}_{0}(x-y$ averaged $\mathrm{K}-\mathrm{Q}$ excitons $),{ }^{42}$ the extracted total density should deviate by less than roughly one- third from the actual pair density in the mixture of different states.

Overall, our findings are consistent with the recent experimental $^{31}$ and theoretical ${ }^{48}$ studies on the exciton formation in TMDC monolayers. In particular, the interband pump-probe data of ref 31 have been explained by formation times on the order of 0.3 ps across several material systems and for a broad range of excitation energies, up to $2.38 \mathrm{eV}$ for $\mathrm{WSe}_{2}$, corresponding well to our findings. With respect to the theory, high above-bandgap excitation in our experiment enables absorption channels across the Brillouin zone and is expected to lead to rich, nontrivial dynamics on ultrashort time scales in contrast to carrier injection close to the free particle band edge at the $\mathrm{K}$ and $\mathrm{K}^{\prime}$ points considered in ref 48 .

In summary, we have studied the ultrafast intraband response of optically resonantly and nonresonantly pumped $\mathrm{WSe}_{2}$ monolayers. Field-resolved detection of the spectrally broad mid-IR probe pulse allowed us to directly monitor the individual population dynamics of the electron-hole plasma and the tightly bound excitons. Remarkably, we already find an exciton fraction as high as $60 \%$ after the initial relaxation of the photoexcited carriers toward the band edge states within the first few hundreds of femtoseconds. During the subsequent decay of the population on a time scale of several picoseconds, about $70 \%$ of the carriers are bound into excitons and the rest of the photoexcited electrons and holes is still present as a free plasma, indicating long-lived nonequilibrium conditions of the carrier system. The findings are of major importance both for our understanding of the fundamental physics of photoexcited 2D TMDCs and for their potential future applications. On the fundamental side, the clear evidence of a rapid exciton formation implies highly efficient exciton injection even under strongly nonresonant excitation conditions. With respect to applications, the presence of a significant fraction of free charge carriers at comparatively long time scales has major implications for the use of TMDC monolayers in future optoelectronic devices, such as sensors, detectors, and photovoltaics.

\section{ASSOCIATED CONTENT}

\section{S Supporting Information}

The Supporting Information is available free of charge on the ACS Publications website at DOI: 10.1021/acs.nanolett.6b04422.

Optical pump and mid-infrared probe pulses; fieldresolved optical pump/mid-infrared probe spectroscopy; degradation of the $\mathrm{WSe}_{2}$ monolayer; temporal evolution of the field-resolved time-domain data; temporal evolution of the fitting parameters (PDF)

\section{AUTHOR INFORMATION}

\section{Corresponding Authors}

*E-mail: alexey.chernikov@ur.de.

*E-mail: rupert.huber@ur.de.

\section{ORCID}

Philipp Steinleitner: 0000-0001-6808-5547

\section{Notes}

The authors declare no competing financial interest. 


\section{ACKNOWLEDGMENTS}

The authors thank Andor Kormányos, Christoph Pöllmann, and Michael Porer for helpful discussions and Martin Furthmeier for technical assistance. Special thanks go to the authors of ref 42 for providing the band structure data for $\mathrm{WSe}_{2}$. This work was supported by the European Research Council through ERC Grant 305003 (QUANTUMsubCYCLE) and by the Deutsche Forschungsgemeinschaft (DFG) through Research Training Group GK1570 and Project Grant KO3612/1-1. A.C. gratefully acknowledges funding from the Deutsche Forschungsgemeinschaft through the Emmy Noether Programme (CH1672/1-1).

\section{REFERENCES}

(1) Splendiani, A.; Sun, L.; Zhang, Y.; Li, T.; Kim, J.; Chim, C.-Y.; Galli, G.; Wang, F. Nano Lett. 2010, 10, 1271-1275.

(2) Mak, K. F.; Lee, C.; Hone, J.; Shan, J.; Heinz, T. F. Phys. Rev. Lett. 2010, 105, 136805.

(3) Tonndorf, P.; Schmidt, R.; Böttger, P.; Zhang, X.; Börner, J.; Liebig, A.; Albrecht, M.; Kloc, C.; Gordan, O.; Zahn, D. R. T.; et al. Opt. Express 2013, 21, 4908-4916.

(4) Moody, G.; Dass, C. K.; Hao, K.; Chen, C.-H.; Li, L.-J.; Singh, A.; Tran, K.; Clark, G.; Xu, X.; Berghäuser, G.; et al. Nat. Commun. 2015, 6, 8315 .

(5) Xu, X.; Yao, W.; Xiao, D.; Heinz, T. F. Nat. Phys. 2014, 10, 343350.

(6) Wang, G.; Glazov, M. M.; Robert, C.; Amand, T.; Marie, X.; Urbaszek, B. Phys. Rev. Lett. 2015, 115, 117401.

(7) Plechinger, G.; Nagler, P.; Arora, A.; Schmidt, R.; Chernikov, A.; del Águila, A. G.; Christianen, P. C.M.; Bratschitsch, R.; Schüller, C.; Korn, T. Nat. Commun. 2016, 7, 12715.

(8) Wang, Q. H.; Kalantar-Zadeh, K.; Kis, A.; Coleman, J. N.; Strano, M. S. Nat. Nanotechnol. 2012, 7, 699-712.

(9) Britnell, L.; Ribeiro, R. M.; Eckmann, A.; Jalil, R.; Belle, B. D.; Mishchenko, A.; Kim, Y.-J.; Gorbachev, R. V.; Georgiou, T.; Morozov, S. V.; et al. Science 2013, 340, 1311-1314.

(10) Jariwala, D.; Sangwan, V. K.; Lauhon, L. J.; Marks, T. J.; Hersam, M. C. ACS Nano 2014, 8, 1102-1120.

(11) Koppens, F. H. L.; Mueller, T.; Avouris, Ph.; Ferrari, A. C.; Vitiello, M. S.; Polini, M. Nat. Nanotechnol. 2014, 9, 780-793.

(12) Berkelbach, T. C.; Hybertsen, M. S.; Reichman, D. R. Phys. Rev. B: Condens. Matter Mater. Phys. 2013, 88, 045318.

(13) Qiu, D. Y.; da Jornada, F. H.; Louie, S. G. Phys. Rev. Lett. 2013, 111, 216805.

(14) Zhang, C.; Johnson, A.; Hsu, C.; Li, L.; Shih, C. Nano Lett. 2014, 14, 2443-2447.

(15) Ye, Z.; Cao, T.; O’Brien, K.; Zhu, H.; Yin, X.; Wang, Y.; Louie,

S. G.; Zhang, X. Nature 2014, 513, 214-218.

(16) He, K.; Kumar, N.; Zhao, L.; Wang, Z.; Mak, K. F.; Zhao, H.; Shan, J. Phys. Rev. Lett. 2014, 113, 026803.

(17) Chernikov, A.; Berkelbach, T. C.; Hill, H. M.; Rigosi, A.; Li, Y.; Aslan, O. B.; Reichman, D. R.; Hybertsen, M. S.; Heinz, T. F. Phys. Rev. Lett. 2014, 113, 076802.

(18) Ugeda, M. M.; Bradley, A. J.; Shi, S.-F.; da Jornada, F. H.; Zhang, Y.; Qiu, D. Y.; Ruan, W.; Mo, S.-K.; Hussain, Z.; Shen, Z.-X.; et al. Nat. Mater. 2014, 13, 1091-1095.

(19) Poellmann, C.; Steinleitner, P.; Leierseder, U.; Nagler, P.; Plechinger, G.; Porer, M.; Bratschitsch, R.; Schüller, C.; Korn, T.; Huber, R. Nat. Mater. 2015, 14, 889-893.

(20) Zhu, C. R.; Zhang, K.; Glazov, M.; Urbaszek, B.; Amand, T.; Ji, Z. W.; Liu, B. L.; Marie, X. Phys. Rev. B: Condens. Matter Mater. Phys. 2014, 90, 161302 .

(21) Chernikov, A.; Ruppert, C.; Hill, H. M.; Rigosi, A. F.; Heinz, T. F. Nat. Photonics 2015, 9, 466-470.

(22) Kumar, N.; Cui, Q.; Ceballos, F.; He, D.; Wang, Y.; Zhao, H. Phys. Rev. B: Condens. Matter Mater. Phys. 2014, 89, 125427.
(23) Chen, H.; Wen, X.; Zhang, J.; Wu, T.; Gong, Y.; Zhang, X.; Yuan, J.; Yi, C.; Lou, J.; Ajayan, P. M.; et al. Nat. Commun. 2016, 7, 12512.

(24) Mak, K. F.; He, K.; Lee, C.; Lee, G. H.; Hone, J.; Heinz, T. F.; Shan, J. Nat. Mater. 2012, 12, 207-211.

(25) You, Y.; Zhang, X.-X.; Berkelbach, T. C.; Hybertsen, M. S.; Reichman, D. R.; Heinz, T. F. Nat. Phys. 2015, 11, 477-481.

(26) Lagarde, D.; Bouet, L.; Marie, X.; Zhu, C. R.; Liu, B. L.; Amand, T.; Tan, P. H.; Urbaszek, B. Phys. Rev. Lett. 2014, 112, 047401.

(27) Wang, H.; Zhang, C.; Chan, W.; Manolatou, C.; Tiwari, S.; Rana, F. Phys. Rev. B: Condens. Matter Mater. Phys. 2016, 93, 045407.

(28) Zimmermann, J. E.; Mette, G.; Höfer, U. 2016, arxiv $1608.03434 \mathrm{v} 2$.

(29) Robert, C.; Lagarde, D.; Cadiz, F.; Wang, G.; Lassagne, B.; Amand, T.; Balocchi, A.; Renucci, P.; Tongay, S.; Urbaszek, B.; et al. Phys. Rev. B: Condens. Matter Mater. Phys. 2016, 93, 205423.

(30) Selig, M.; Berghäuser, G.; Raja, A.; Nagler, P.; Schüller, C.; Heinz, T. F.; Korn, T.; Chernikov, A.; Malic, E.; Knorr, A. Nat. Commun. 2016, 7, 13279.

(31) Ceballos, F.; Cui, Q.; Bellus, M. Z.; Zhao, H. Nanoscale 2016, 8, 11681-11688.

(32) Huber, R.; Tauser, F.; Brodschelm, A.; Bichler, M.; Abstreiter, G.; Leitenstorfer, A. Nature 2001, 414, 286-289.

(33) Kaindl, R. A.; Carnahan, M. A.; Hägele, D.; Lövenich, R.; Chemla, D. S. Nature 2003, 423, 734-738.

(34) Leinß, S.; Kampfrath, T.; v. Volkmann, K.; Wolf, M.; Steiner, J. T.; Kira, M.; Koch, S. W.; Leitensdorfer, A.; Huber, R. Phys. Rev. Lett. 2008, 101, 246401.

(35) Porer, M.; Leierseder, U.; Ménard, J.-M.; Dachraoui, H.; Mouchliadis, L.; Perakis, I. E.; Heinzmann, U.; Demsar, J.; Rossnagel, K.; Huber, R. Nat. Mater. 2014, 13, 857-861.

(36) Ménard, J.-M.; Poellmann, C.; Porer, M.; Leierseder, U.; Galopin, E.; Lemaitre, A.; Amo, A.; Bloch, J.; Huber, R. Nat. Commun. 2014, 5, 4648.

(37) Cha, S.; Sung, J. H.; Sim, S.; Park, J.; Heo, H.; Jo, M.-H.; Choi, H. Nat. Commun. 2016, 7, 10768.

(38) Kubouchi, M.; Yoshioka, K.; Shimano, R.; Mysyrowicz, A.; Kuwata-Gonokami, M. Phys. Rev. Lett. 2005, 94, 016403.

(39) Huber, R.; Schmid, B. A.; Shen, Y. R.; Chemla, D. S.; Kaindl, R. A. Phys. Rev. Lett. 2006, 96, 017402.

(40) Castellanos-Gomez, A.; Buscema, M.; Molenaar, R.; Singh, V.; Janssen, L.; van der Zant, H. S. J.; Steele, G. A. 2D Mater. 2014, 1, 011002.

(41) Li, Y.; Chernikov, A.; Zhang, X.; Rigosi, A.; Hill, H. M.; van der Zande, A. M.; Chenet, D. A.; Shih, E.-M.; Hone, J.; Heinz, T. F. Phys. Rev. B: Condens. Matter Mater. Phys. 2014, 90, 205422.

(42) Kormányos, A.; Burkard, G.; Gmitra, M.; Fabian, J.; Zólyomi, V.; Drummond, N. D.; Fal'ko, V. 2D Mater. 2015, 2, 022001.

(43) Kaindl, R. A.; Hängele, D.; Carnahan, M. A.; Chemla, D. S. Phys. Rev. B: Condens. Matter Mater. Phys. 2009, 79, 045320.

(44) Singh, A.; Moody, G.; Tran, K.; Scott, M. E.; Overbeck, V.; Berghäuser, G.; Schaibley, J.; Seifert, E. J.; Pleskot, D. N.; Gabor, M.; et al. Phys. Rev. B: Condens. Matter Mater. Phys. 2016, 93, 041401.

(45) Pötz, W.; Kocevar, P. Phys. Rev. B: Condens. Matter Mater. Phys. 1983, 28, 7040 .

(46) Terrones, H.; Del Corro, E.; Feng, S.; Poumirol, J. M.; Rhodes, D.; Smirnov, D.; Pradhan, N. R.; Lin, Z.; Nguyen, M. A. T.; Elías, A. L.; et al. Sci. Rep. 2014, 4, 4215.

(47) Zhang, X.-X.; You, Q.; Yang, S.; Zhao, F.; Heinz, T. F. Phys. Rev. Lett. 2015, 115, 257403.

(48) Thilagam, A. J. Appl. Phys. 2016, 120, 124306. 